\title{
Minimal geometric deformation of Yang-Mills-Dirac stellar configurations
}

\author{
Roldão da Rocha®* \\ Center of Mathematics, Federal University of ABC, 09210-580 Santo André, Brazil
}

(Received 30 March 2020; accepted 22 June 2020; published 2 July 2020)

\begin{abstract}
The method of minimal geometric deformation (MGD) is used to derive static, strongly gravitating, spherically symmetric, compact stellar distributions that are solutions of the Yang-Mills-Einstein-Dirac coupled field equations on fluid membranes with finite tension. Their solutions characterize MGD YangMills-Dirac stars, whose mass has order of the Chandrasekhar mass, once the range of both the fermionic self-interaction and the Yang-Mills coupling constants is suitably chosen. Physical features of MGD YangMills-Dirac stars are then discussed and their Arnowitt-Deser-Misner masses are derived, as a function of the fermion coupling constant, the finite brane tension, and the Yang-Mills running parameter.
\end{abstract}

DOI: $10.1103 /$ PhysRevD.102.024011

\section{INTRODUCTION}

The minimal geometricci deformation (MGD) and the MGD-decoupling methods consist of successful procedures that can engender analytical solutions of the brane Einstein's effective field equations in AdS/CFT and its membrane paradigm [1-5]. Our Universe, the codimension-1 brane with intrinsic tension, is embedded in a bulk [6,7]. MGD procedures can be implemented when one deforms already known solutions in general relativity (GR). Standard gravity in GR consists of the rigid brane, with infinite tension, limit. The brane tension is interpreted as the finite vacuum energy. Since recent phenomenological data imply a finite brane tension $(\sigma)$ with the most precise observational bound $\sigma \gtrsim 2.81 \times$ $10^{-6} \mathrm{GeV}^{4}$ [8], the MGD method represents a realistic procedure to derive and analyze compact stellar distributions, complying with current cosmological observations. The finite brane tension drives the ways to deform the Schwarzschild solution of the Einstein field equations [9-13]. The cosmic microwave background (CMB) anisotropic aspects, showed by WMAP, forced the fluid brane tension to satisfy the Eötvös law, asserting that the surface tension of a membrane is proportional to its temperature [14-16]. Regarding models describing cosmic inflation, the tension of the brane must have fluctuated, as the temperature of the Universe decreased down to the current value $\sim 2.7 \mathrm{~K}[16]$.

roldao.rocha@ufabc.edu.br

Published by the American Physical Society under the terms of the Creative Commons Attribution 4.0 International license. Further distribution of this work must maintain attribution to the author(s) and the published article's title, journal citation, and DOI. Funded by SCOAP ${ }^{3}$.
MGD provides analytical solutions of strongly gravitating, compact star distributions and black holes as well $[2,4,5,17,18]$. MGD is ruled by a running parameter, proportional to the inverse of the brane tension, with recent observational/experimental constraints [8,19,20]. MGD and its extensions have been employed to study the configurational stability of stellar distributions $[8,20]$, whose observational signature in LIGO and eLISA was proposed in Refs. [21,22]. Einstein-Klein-Gordon configurations, using MGD gravitational decoupling, were studied in Ref. [23]. MGD analogs in the laboratory were proposed [24] and the Gregory-Laflamme instability phenomenon was shown to be precluded in MGD black strings with critical masses [25]. The MGD procedure and the MGD gravitational decoupling method have also been investigated and used in Refs. [26-37], encompassing the analysis and scrutiny of anisotropic configurations [38-50]. Higher derivative terms in the gravitational decoupling were studied in Refs. [51,52]. In addition, the $(2+1)$ dimensional version of MGD gravitational decoupling, with several applications, was discussed in Refs. [53-56]. Still, new nuances of the MGD in AdS/CFT have been paved by the study of the holographic entanglement entropy of MGD solutions [57].

In this work, MGD Yang-Mills-Dirac stellar configurations will be derived as solutions of a Yang-Mills-EinsteinDirac coupled system of field equations on a fluid brane with finite tension. We will analyze the finite brane tension influence on the compact stellar configurations. Without taking into account Yang-Mills fields, solutions of the Einstein-Dirac coupled system consist of Dirac stellar distributions, driven by nonlinear spinor fields [58,59]. Afterward, Maxwell, Yang-Mills, and even Proca fields were coupled to the Einstein-Dirac coupled system, always in the GR, $\sigma \rightarrow \infty$ limit [60-62]. MGD Dirac stars were 
proposed and studied in Ref. [63], with astonishing physical consequences that arise from the brane tension finite value. The Einstein-Dirac coupled system of field equations have static and analytical solutions [64,65]. Self-gravitating systems of spin- $1 / 2$ fermionic fields were also studied in Refs. $[66,67]$. The main aim of this work is to study MGD compact stellar configurations as solutions of the effective Yang-Mills-Einstein-Dirac coupled system of field equations on a fluid brane with finite tension.

Among the possible Yang-Mills fields that can be employed, we will approach SU(2) monopolelike fields. In fact, Refs. [60-62,68] already studied static, spherically symmetric configurations arising from Yang-Mills equations, however, only in the GR limit of an infinitely rigid brane, with $\sigma \rightarrow \infty$. Hence, it is natural to question, in a realistic model that complies with recent observational data, how the finite tension of the brane can drive new MGD solutions of the Yang-Mills-Einstein-Dirac system. These solutions will be shown to form compact MGD Yang-Mills-Dirac stellar configurations whose physical properties will be scrutinized. Besides the MGD procedure, a MGD-decoupling-like technique will also be utilized to construct a more reliable stress-energy brane tensor, induced by nonlinear spinor fields. For fermion masses that are orders of magnitude smaller than the Planck mass, the MGD-decoupled solutions will be shown to characterize MGD Yang-Mills-Dirac stars, with mass of the order of the Chandrasekhar mass. Self-interacting spinor fields will then be employed to investigate the Yang-Mills-Dirac stars' main physical features, whose Arnowitt-Deser-Misner $(\mathrm{ADM})$ mass is a function of the fermion self-interaction and Yang-Mills coupling constants.

This paper is organized as follows: Sec. II is dedicated to reviewing the MGD derivation as a complete method to deform the Schwarzschild solution and to describe realistic stellar distributions on finite tension branes. In Sec. III, the MGD Yang-Mills-Einstein-Dirac coupled system of field equations on fluid branes with finite tension is numerically solved, analyzed, and discussed. MGD Yang-Mills-Dirac stellar configurations, whose mass has order of the Chandrasekhar mass, are studied and discussed. The fermionic self-interaction and Yang-Mills coupling constants ranges are physically bounded. Section IV is devoted to conclusions and important perspectives.

\section{THE MGD AND MGD-DECOUPLING PROTOCOLS}

The MGD procedure, founded by Ovalle, is constructed to derive high energy scale corrections to GR [2,5,12]. Fluid branes have a variable tension that emulates cosmological evolution $[14,16]$. The extended MGD derived the most rigorous brane tension bound, $\sigma \gtrsim 2.81 \times 10^{-6} \mathrm{GeV}^{4}$ [8].

Since the codimension-1 brane is embedded in the bulk, the extrinsic curvature, defined as the Lie derivative of the metric tensor, plays a prominent role in deriving equations of motion. As the bulk Riemann tensor can be written in terms of the brane Riemann tensor via the Gauss-Codazzi equation, the effective Einstein equations on the brane read

$$
\mathrm{G}_{\mu \nu}=\Lambda_{b} g_{\mu \nu}+\mathfrak{T}_{\mu \nu}
$$

where $8 \pi G=1$ is adopted, with $G$ being Newton's constant on the brane, $\mathrm{G}_{\mu \nu}$ represents the Einstein's tensor, and $\Lambda_{b}$ denotes the brane cosmological running parameter. One can cleave the stress-energy tensor in Eq. (1) into [69]

$$
\mathfrak{T}_{\alpha \beta}=T_{\alpha \beta}+\mathfrak{E}_{\alpha \beta}+\sigma^{-1} S_{\alpha \beta}+L_{\alpha \beta}+P_{\alpha \beta} .
$$

The first term, $T_{\alpha \beta}$, is the stress-energy tensor that represents brane matter and energy, eventually including dark matter and dark energy, whereas the electric part of the bulk Weyl tensor is denoted by $\mathfrak{E}_{\alpha \beta}$. The tensor $\mathfrak{E}_{\alpha \beta}$ is nonlocal and depends on $\sigma^{-1}$, vanishing in the general-relativistic limit. Splitting $\mathfrak{E}_{\alpha \beta}$ into traceless transverse $\left(\mathfrak{E}_{\alpha \beta}^{\top}\right)$ and longitudinal $\left(\mathfrak{F}_{\alpha \beta}^{L}\right)$ components, Shiromizu et al. [69] showed that $\mathfrak{F}_{\alpha \beta}^{L}$ contains solely brane matter terms. Therefore, the resulting equations of motion are closed when $\mathfrak{E}_{\alpha \beta}^{\mathrm{T}}=0$ since this term contains Kaluza-Klein bulk gravitons, whose backreaction and interaction with brane matter influence the equations of motion. The $S_{\alpha \beta}$ is a tensor that consists of quadratic terms involving the stressenergy tensor arising from the extrinsic curvature terms in the projected Einstein tensor. It encodes local corrections and is given by $[69,70]$

$$
S_{\alpha \beta}=\frac{T}{3} T_{\alpha \beta}-T_{\alpha \mu} T_{\beta}^{\mu}+\frac{g_{\alpha \beta}}{6}\left[3 T_{\mu \rho} T^{\mu \rho}-T^{2}\right],
$$

where $T=T_{\alpha}^{\alpha}$ denotes the trace of $T_{\alpha \beta}$. Its intensity is smaller than $\mathfrak{E}_{\alpha \beta}$ [71]. In addition, $L_{\alpha \beta}$ encodes the geometry of how the brane bends into the bulk, whereas $P_{\alpha \beta}$ comprises stringy bosonic and fermionic fields in the bulk [16,71].

Hence, the effective Einstein field equations (1) encode corrections, due mainly to the bulk Weyl fluid [69]. The Weyl fluid flow can be put through the bulk Weyl tensor projected onto the brane,

$$
\mathfrak{E}_{\alpha \beta}=-\frac{6}{\sigma}\left[\mathfrak{U}\left(u_{\alpha} u_{\beta}+\frac{1}{3} h_{\alpha \beta}\right)+Q_{(\alpha} u_{\beta)}+\mathfrak{P}_{\alpha \beta}\right],
$$

where $h_{\alpha \beta}$ denotes the projector operator that is orthogonal to the Weyl fluid flow velocity $u^{\alpha}$. In addition,

$$
\mathfrak{U}=-\frac{1}{6} \sigma \mathfrak{F}_{\alpha \beta} u^{\alpha} u^{\beta}
$$

is the effective energy density, sometimes playing the role of dark radiation [17], whereas 


$$
\mathfrak{P}_{\alpha \beta}=-\frac{1}{6} \sigma\left(h_{(\alpha}^{\mu} h_{\beta)}^{\rho}-\frac{1}{3} h^{\mu \rho} h_{\alpha \beta}\right) \mathfrak{F}_{\mu \rho}
$$

is the nonlocal anisotropic stress tensor. The effective nonlocal energy flux,

$$
Q_{\alpha}=-\frac{1}{6} \sigma h^{\mu \rho} \mathfrak{夭}_{\rho \alpha} u_{\mu},
$$

has the bulk free gravitational field as the source.

Compact stars are solutions of the system of equations (1), with metric

$$
d s^{2}=-A(r) d t^{2}+\frac{1}{B(r)} d r^{2}+r^{2} d \vartheta^{2}+r^{2} \sin ^{2} \vartheta d \varphi^{2} .
$$

One can usually write

$$
A(r)=e^{\nu(r)}, \quad B(r)=e^{\chi(r)}
$$

as more concise notation for what follows. This spherically symmetric setup allows Eqs. (6) and (7) to be expressed, respectively, as $\mathfrak{P}_{\alpha \beta}=\mathfrak{P}\left(u_{\alpha} u_{\beta}+h_{\alpha \beta} / 3\right)$ and $Q_{\alpha}=0$, where $\mathfrak{P}=\mathfrak{P}_{\alpha}{ }^{\alpha}$. Correspondingly, the stress-energy tensor that represents brane matter and energy can be chosen as the one of a perfect fluid, $T_{\alpha \beta}=(\rho+p) u_{\alpha} u_{\beta}-p g_{\alpha \beta}$, with $u^{\alpha}=\delta_{0}^{\alpha} / \sqrt{A}$, whereas $g_{\alpha \beta}$ denotes the metric components. The MGD and MGD-decoupling methods, together with their generalizations, can produce analytical solutions of system (1) and (2) $[1,2,4,5]$, including the inner solutions concerning star distributions of radius $R$, given by

$$
R=\frac{\int_{0}^{\infty} d \mathrm{rr}^{3} \varrho(\mathrm{r})}{\int_{0}^{\infty} d \mathrm{r}^{2} \varrho(\mathrm{r})},
$$

where $Q(r)$ represents the density of the star distribution [2]. In what follows in this section, both procedures will be discussed.

\section{A. MGD procedure}

The effective Einstein equations on the brane (1) are equivalently written as

$$
\begin{gathered}
e^{\chi}=1-\frac{8 \pi G}{c^{4} r} \int_{0}^{r} \mathfrak{r}^{2}\left[\varrho+\frac{1}{\sigma}\left(\frac{\varrho^{2}}{2}+\frac{3 \mathfrak{U} c^{8}}{32 \pi^{2} G^{2}}\right)\right] d \mathfrak{x}, \\
\frac{\mathfrak{P} c^{4}}{8 \pi G \sigma}=\frac{1}{6}\left(G_{1}{ }^{1}-G_{2}{ }^{2}\right), \\
\frac{3 \mathfrak{U} c^{8}}{32 \pi^{2} G^{2}}=-3\left(\frac{\varrho^{2}}{2}+\varrho p\right)+\frac{c^{4} \sigma}{8 \pi G}\left(2 G_{2}{ }^{2}+G_{1}{ }^{1}\right)-3 p \sigma, \\
p^{\prime}=-\frac{\nu^{\prime}}{2}(\varrho+p),
\end{gathered}
$$

where

$$
\begin{gathered}
G_{1}{ }^{1}=-\frac{1}{r^{2}}+e^{-\chi}\left(\frac{1}{r^{2}}+\frac{\nu^{\prime}}{r}\right), \\
G_{2}{ }^{2}=\frac{1}{4} e^{-\chi}\left[2 \nu^{\prime \prime}+\nu^{\prime 2}-\chi^{\prime} \nu^{\prime}+\frac{2\left(\nu^{\prime}-\chi^{\prime}\right)}{r}\right]
\end{gathered}
$$

for $f^{\prime}=d f / d r$. GR is recovered when $\sigma^{-1} \rightarrow 0$ and with Eq. (14).

Anisotropy immediately follows when one takes Eqs. (11)-(13) and writes the effective density $(\stackrel{\circ}{\varrho})$, the radial pressure $\left(\stackrel{\circ}{p}_{r}\right)$, and the tangential pressure $\left(\stackrel{\circ}{p}_{t}\right)$, respectively, as [5]

$$
\begin{gathered}
\stackrel{\circ}{\varrho}=\varrho+\frac{1}{\sigma}\left(\frac{\varrho^{2}}{2}+\frac{3 \mathfrak{U} c^{4}}{32 \pi^{2} G^{2}}\right), \\
\stackrel{\circ}{p}_{r}=p+\frac{1}{\sigma}\left(\frac{\varrho^{2}}{2}+\varrho p+\frac{\mathfrak{U} c^{4}}{32 \pi^{2} G^{2}}\right)+\frac{\mathfrak{P} c^{4}}{16 \pi^{2} G^{2} \sigma}, \\
\stackrel{\circ}{p}_{t}=p+\frac{1}{\sigma}\left(\frac{\varrho^{2}}{2}+\varrho p+\frac{\mathfrak{U} c^{4}}{32 \pi^{2} G^{2}}\right)-\frac{\mathfrak{P} c^{4}}{32 \pi^{2} G^{2} \sigma} .
\end{gathered}
$$

Anisotropy on the stellar configuration, caused by the bulk Weyl fluid, then reads

$$
\Delta=\stackrel{\circ}{p}_{r}-\stackrel{\circ}{p}_{t}=\frac{3 \mathfrak{P} c^{4}}{32 \pi^{2} G^{2} \sigma} .
$$

Equation (17) asserts that the MGD effective stellar density is given by the sum of the stellar density and a dark radiation term involving $\mathfrak{U}$, as given in Eq. (5), mediated by the brane electric part of the Weyl tensor. The stellar configuration central density is therefore changed by the term $\frac{1}{\sigma}\left(\frac{Q^{2}}{2}+\frac{3 \mathfrak{H} c^{4}}{32 \pi^{2} G^{2}}\right)$. This occurs by bulk effects and also by the brane tension finiteness $\sigma \gtrsim 2.81 \times 10^{-6} \mathrm{GeV}^{4}$ [8].

Equations (11)-(14) consist of a coupled system of field ordinary differential equations (ODEs) on the brane. With the system, braneworld varieties of GR solutions can be constructed using MGD. In fact, bulk gravity yields a minimal geometric deformation ${ }^{1} \kappa(r)$ in the radial metric component, given by [9]

$$
e^{-\chi(r)}=\mu(r)+\kappa(r),
$$

where

\footnotetext{
${ }^{1}$ It is minimal, as every other source inducing the geometric deformation has been removed, aside from those yielded by density and pressure in stellar configurations.
} 


$$
\mu(r)= \begin{cases}1-\frac{1}{G^{2} r} \int_{0}^{r} \mathrm{r}^{2} \varrho(\mathrm{r}) d \mathrm{r}, & r \leq R \\ 1-\frac{2 G M_{0}}{c^{2} r}, & r>R\end{cases}
$$

Alternatively, both ranges in Eq. (22) can be encoded as

$$
\mu(r)=1-\frac{2 G m(r)}{c^{2} r},
$$

which contains the mass function $m(r)$.

When one takes the apparently straightforward way to derive a solution to the coupled system (11)-(14), substituting Eq. (13) into the original form of Eq. (11), which is the field equation

$\frac{8 \pi G}{c^{4}}\left[\varrho+\frac{1}{\sigma}\left(\frac{\varrho^{2}}{2}+\frac{3 \mathcal{U}}{32 \pi^{2} G^{2}}\right)\right]=\frac{1}{r^{2}}-e^{-\chi}\left(\frac{1}{r^{2}}-\frac{\chi^{\prime}}{r}\right)$,

yields an ODE to the geometric function $e^{-\chi}$ given by

$$
\begin{aligned}
e^{-\chi} & \left(\frac{2 r^{2} \nu^{\prime \prime}(r)+r^{2} \nu^{\prime 2}(r)+4 r \nu^{\prime}(r)+4}{r^{2} \nu^{\prime}(r)+4 r}-\chi^{\prime}\right) \\
= & \frac{4}{r\left(\nu^{\prime} r+4\right)}-\frac{8 \pi G}{c^{4}} \frac{\left(\varrho-3 p-\frac{\varrho}{\sigma}(\varrho+3 p)\right)}{\left(\nu^{\prime} / 2+2 / r\right)} .
\end{aligned}
$$

The formal solution of Eq. (25) reads

$$
\begin{aligned}
e^{-\chi}= & e^{-I}\left(\int _ { 0 } ^ { r } \frac { e ^ { I } } { \frac { \nu ^ { \prime } } { 2 } + \frac { 2 } { \mathfrak { r } } } \left[\frac{2}{\mathfrak{x}^{2}}-\frac{8 \pi G}{c^{4}}\right.\right. \\
& \left.\left.\times\left(\varrho-3 p-\frac{\varrho}{\sigma}(\varrho+3 p)\right)\right] d \mathfrak{x}+\beta\right),
\end{aligned}
$$

where $\beta$ is a parameter that depends on the brane tension and

$$
I=\int_{0}^{r} \frac{2 \mathfrak{r}^{2} \nu^{\prime \prime}(\mathfrak{r})+\mathfrak{r}^{2} \nu^{\prime 2}(\mathfrak{r})+4 \mathfrak{r} \nu^{\prime}(\mathfrak{r})+4}{\mathfrak{r}^{2} \nu^{\prime}(\mathfrak{r})+4 \mathfrak{r}} d \mathfrak{r} .
$$

Therefore, when a solution $\{p, \varrho, \nu\}$ to Eq. (14) is found, one can derive $\chi, \mathfrak{P}$, and $\mathfrak{U}$ using Eqs. (26), (12), and (13), respectively.

Matching Eq. (26), Ovalle [3] then showed that the MGD function in Eq. (21) is given by

$\kappa(r)=e^{-I}\left(\beta+\int_{0}^{r} \frac{2 \mathfrak{x} e^{I}}{\mathfrak{r} \nu^{\prime}+4}\left[L+\frac{1}{G^{2} \sigma}\left(\varrho^{2}+3 p \varrho\right)\right]\right) d \mathfrak{x}$.

The function $L(r)=L(p(r), \varrho(r), \nu(r))$, given by

$$
\begin{aligned}
L(p, \varrho, \nu)= & {\left[\mu^{\prime}\left(\frac{\nu^{\prime}}{2}+\frac{1}{r}\right)+\mu\left(\nu^{\prime \prime}+\frac{\nu^{\prime 2}}{2}+\frac{2 \nu^{\prime}}{r}+\frac{1}{r^{2}}\right)-\frac{1}{r^{2}}\right] } \\
& -\frac{24 \pi G}{c^{4}} p
\end{aligned}
$$

also encodes the brane anisotropy, induced by gravity in the bulk on the set $p(r), \varrho(r)$, and $\nu(r)$. For interior solutions, the condition $\beta(\sigma)=0$ must be imposed to avoid singular solutions at the center $r=0$ of the stellar configuration. However, in the region $r>R$ where there is a Weyl fluid surrounding the stellar distribution, the function $\beta(\sigma)$ is not zero, in general.

As the finite brane tension has the strictest bound $\sigma \gtrsim 2.81 \times 10^{-6} \mathrm{GeV}^{4}$ [8], the scalar field $\beta=\beta(\sigma)$ will be shown to depend on the inverse of $\sigma$, in full compliance with phenomenological data. Hence, the generalrelativistic limit yields $\lim _{\sigma \rightarrow \infty} \beta(\sigma)=0$ for outer, $r>R$ solutions. The geometric deformation $\kappa(r)$ in the vacuum $(p=0=\varrho)$, denoted by $h^{*}(r)$ in what follows, is minimal, reading $[2,4]$

$$
h^{*}(r)=\beta(\sigma) e^{-I} .
$$

The radial metric component in Eq. (21) then becomes

$$
\frac{1}{B(r)}=e^{\chi(r)}=1-\frac{2 M_{0}}{r}+\beta(\sigma) e^{-I} .
$$

Matching conditions among the surface of the stellar configuration, $r=R$, its interior, $r<R$, and its exterior, $r>R$, drives the final metric solutions for each of these regions. Taking into account Eqs. (22) and (23), with $r<R$, the inner MGD metric reads [72]

$d s^{2}=-e^{\nu^{-}(r)} d t^{2}+\frac{1}{1-\frac{2 G \dot{\circ}(r)}{c^{2} r}} d r^{2}+r^{2}\left(d \vartheta^{2}+\sin ^{2} \vartheta d \varphi^{2}\right)$,

where the interior mass function reads

$$
\stackrel{\circ}{m}(r)=m(r)-\frac{r}{2} \kappa^{*}(r),
$$

with $m(r)$ given by the standard GR formulas (22) and (23) and $\kappa^{*}$ corresponding to the MGD in Eq. (28), with $L=0$. The inner metric (32) must be then matched with an outer geometry, with $p^{+}=Q^{+}=0$.

Junction conditions match the inner MGD metric (32) to the outer solution. The Weyl fluid that circumscribe the brane can be represented by the outer pressure and the bulk Weyl scalar, respectively, given by the condensed forms

$$
\mathfrak{P}^{+}(r)=-\frac{\left(1-\frac{4 G M}{3 c^{2} r}\right) \beta(\sigma)}{9 G^{2} \sigma r^{3}\left(1-\frac{3 G M}{2 c^{2} r}\right)^{2}},
$$




$$
\mathfrak{U}^{+}(r)=\frac{M \beta(\sigma)}{12 G c^{2} \sigma r^{4}\left(1-\frac{3 G M}{2 c^{2} r}\right)^{2}}
$$

In this case, $p(r)=0=Q(r)$ for $r>R$, and the outer solution metric reads $[4,72]$

$d s^{2}=-e^{\nu^{+}(r)} d t^{2}+\frac{d r^{2}}{1-\frac{2 G M(\sigma)}{c^{2} r}+h^{*}(r)}+r^{2}\left(d \vartheta^{2}+\sin ^{2} \vartheta d \varphi^{2}\right)$.

Matching conditions at the stellar surface $r=R$ yield [4]

$$
\begin{gathered}
\nu^{-}(R)=\nu^{+}(R)=\log \left(1-\frac{2 G M}{c^{2} R}\right), \\
\frac{2 G}{R}\left(M-M_{0}\right)=h^{*}(R)-\kappa^{*}(R) .
\end{gathered}
$$

It is worth emphasizing that in Eq. (38) the effective mass is given by $M=M_{0}+\mathcal{O}\left(\sigma^{-1}\right)$, where terms of order $\mathcal{O}\left(\sigma^{-2}\right)$ on are dismissed due to the phenomenological lower bound for the brane tension $\sigma \approx 2.81 \times 10^{-6} \mathrm{GeV}^{4}$ [8]. The Weyl fluid implies the matching condition [10]

$$
\begin{aligned}
& \sigma p(R)+\left[\frac{\varrho^{2}(R)}{2}+\varrho(R) p(R)+2 G^{4}\left(\mathfrak{U}^{-}(R)-\mathfrak{U}^{+}(R)\right)\right] \\
& +4 G^{4}\left(\mathfrak{P}^{-}(R)-\mathfrak{P}^{+}(R)\right)=0,
\end{aligned}
$$

where $\kappa(R)^{ \pm}=\lim _{r \rightarrow R^{ \pm}} \kappa(r)$.

The Schwarzschild-like solution, $e^{\nu_{\mathrm{Sch}}(r)}=e^{-\chi_{\mathrm{Sch}}(r)}=$ $1-\frac{2 G M}{c^{2} r}$ can then be substituted into Eq. (30), implying that

$$
h^{*}(r)=-\frac{2\left(1-\frac{2 G M}{c^{2} r}\right)}{r\left(r-\frac{3 G M}{2 c^{2}}\right)} \beta(\sigma) .
$$

The function $\beta(\sigma)$ can thus be read off from Eq. (39), also equivalently expressed as [4]

$$
R^{2} p(R)+G^{2} \kappa^{*}(R)\left(R \nu^{\prime}(R)+1\right)=-h^{*}(R) .
$$

The outer deformation $h^{*}(r)$, at $r=R$, has a negative value. Hence, the MGD horizon, $r_{\mathrm{MGD}}=2 G M / c^{2}$, remains closer to the star center if compared to the Schwarzschild one, $r_{\mathrm{Sch}}=2 G M_{0} / c^{2}$, since $M=M(\sigma)=$ $M_{0}+\mathcal{O}\left(\sigma^{-1}\right)[4,17]$.

Equations (40) and (41) imply that [4]

$$
\beta(\sigma)=\frac{c^{2} R-\frac{3 G M}{2}}{c^{2} R-2 G M}\left[\left(R^{2} \nu^{\prime}(R)+R\right) G \kappa^{*}(R)+R^{3} p(R)\right] .
$$

The function $\beta(\sigma)$ can be derived when one remembers that [2] $\kappa^{*}(r)=\frac{4 d l(r)}{49 \sigma \pi} y$, where $y$ is a numerical value [4], $a=\frac{\sqrt{57}-7}{2 R^{2}}$, and $\iota^{-1}(r)=\left(1+a r^{2}\right)^{3}\left(1+3 a r^{2}\right)$ for $\nu^{\prime}(r)=$ $\frac{8 a r}{1+a r^{2}}$. The form of $\kappa(R)$ yields $[2,4]$

$$
\beta(\sigma)=\frac{y}{\sigma R}\left(\frac{c^{2} R-\frac{3 G M_{0}}{2}}{c^{2} R-2 G M_{0}}\right) \equiv \frac{a_{0}}{\sigma} .
$$

The MGD exterior metric can then be written as $[2,4]$

$$
\begin{aligned}
& A(r)=e^{\nu_{\mathrm{Sch}}(r)}=1-\frac{2 G M}{c^{2} r}, \\
& B(r)=\left[1-\frac{2 G M}{c^{2} r}\right]\left(\frac{\mathfrak{l}}{r-\frac{3 G M}{2 c^{2}}}+1\right),
\end{aligned}
$$

where

$$
\mathfrak{l}=\frac{a_{0}}{\sigma} \frac{R-\frac{3 G M}{2 c^{2}}}{R-\frac{2 G M}{c^{2}}}
$$

In the general-relativistic limit $\sigma \rightarrow \infty$, the Schwarzschild metric is recovered from the MGD metric. Casadio et al. [19] derived, employing classical tests of GR, the lower bound $\left|\frac{a_{0}}{\sigma}\right| \lesssim 2.89 \times 10^{-11}$. Therefore, when one reinstates it in Eq. (29) implies that

$$
|\mathfrak{l}| \lesssim 6.26 \times 10^{-4} \mathrm{~m}
$$

for solar size stellar configurations. Varying the mass in Eq. (29) also modifies the star surface radius R, and hence the functions in Eq. (42), accordingly. In fact, Cavalcanti et al. [73] applied the MGD procedure to gravitational lensing effects, where the Sagittarius $A^{*}$ black hole, with mass $M=(4.31 \pm 0.38) \times 10^{6} M_{\odot}$, was considered. The observational value of $\mathfrak{l}=0.0637 \mathrm{~m}$ was then obtained in Ref. [73].

\section{B. MGD-decoupling method}

The gravitational decoupling method asserts that Einstein's field equations read [1]

$$
R_{\mu \nu}-\frac{1}{2} R g_{\mu \nu}=\frac{8 \pi G}{c^{4}} T_{\mu \nu}^{\text {decoupling }}
$$

where

$$
T_{\mu \nu}^{\text {decoupling }}=T_{\mu \nu}^{\text {perfect fluid }}+T_{\mu \nu}^{\text {sources }}
$$

The stress-energy tensor is given by

$$
T_{\mu \nu}^{\text {perfect fluid }}=(\rho+p) u_{\mu} u_{\nu}-p g_{\mu \nu} .
$$

One can consider any contribution from anisotropic gravitational sources $\theta_{\mu \nu}$, modified by a parameter $\zeta$, that drives the intensity of this source as 


$$
T_{\mu \nu}^{\text {sources }}=\zeta \theta_{\mu \nu} .
$$

The $\zeta$ parameter is, in general, inversely proportional to the brane tension $\sigma$ in such a way that the GR limit $\sigma \rightarrow \infty$ yields $\zeta \rightarrow 0$ [1]. Hence, Eq. (48) yields

$$
T_{\mu \nu}^{\text {decoupling }}=(\rho+p) u_{\mu} u_{\nu}-p g_{\mu \nu}+\zeta \theta_{\mu \nu} .
$$

Besides, the conservation law

$$
\nabla^{\nu} T_{\mu \nu}^{\text {decoupling }}=0
$$

follows from the Bianchi identity.

As an alternative method, the MGD decoupling is regarded here by taking into account not necessarily the same metric (8) and (9) but instead, in general, another one,

$d s^{2}=-e^{\nu(r)} d t^{2}+e^{-\chi(r)} d r^{2}+r^{2} d \vartheta^{2}+r^{2} \sin ^{2} \vartheta d \varphi^{2}$,

satisfying the coupled system of Einstein's field equations,

$$
\begin{aligned}
& \frac{8 \pi G}{c^{4}}\left(\rho+\zeta \theta_{0}^{0}\right)=\frac{1}{r^{2}}-e^{-\chi}\left(\frac{1}{r^{2}}-\frac{\chi^{\prime}}{r}\right), \\
& \frac{8 \pi G}{c^{4}}\left(\zeta \theta_{1}{ }^{1}-p\right)=\frac{1}{r^{2}}-e^{-\chi}\left(\frac{1}{r^{2}}-\frac{\nu^{\prime}}{r}\right),
\end{aligned}
$$

$\frac{8 \pi G}{c^{4}}\left(\zeta \theta_{2}^{2}-p\right)=-\frac{e^{-\chi}}{4}\left(2 \nu^{\prime \prime}+\nu^{\prime 2}-\chi^{\prime} \nu^{\prime}+2 \frac{\nu^{\prime}-\chi^{\prime}}{r}\right)$.

Equation (52) then reads

$$
\begin{aligned}
p^{\prime} & +\frac{\nu^{\prime}}{2}(\rho+p)-\zeta\left(\theta_{1}^{1}\right)^{\prime}+\frac{\nu^{\prime} \zeta}{2}\left(\theta_{0}^{0}+\theta_{1}^{1}\right) \\
& +\frac{2 \zeta}{r}\left(\theta_{2}^{2}-\theta_{1}^{1}\right)=0 .
\end{aligned}
$$

Also, the effective density and the effective isotropic and tangential pressures are given, respectively, by

$$
\begin{gathered}
\rho_{\text {effective }}^{\mathrm{radial}}=\rho+\zeta \theta_{0}{ }^{0}, \\
p_{r}=p-\zeta \theta_{1}{ }^{1}, \\
p_{t}=p-\zeta \theta_{2}{ }^{2} .
\end{gathered}
$$

Anisotropy induced by the $\theta_{\mu \nu}$ source can be defined as

$$
\Delta=p_{t}-p_{r}=\zeta\left(\theta_{2}^{2}-\theta_{1}^{1}\right),
$$

emulating that in Eq. (20), here for the MGD-decoupling method. Ovalle [1] showed that the standard MGD can be derived as a particular case of the MGD decoupling, which will be illustrated in this subsection.
To solve the coupled system (54)-(60), the MGD procedure plays a prominent role in the decoupling procedure [1]. For it, a perfect fluid, with energy density $\rho$ and pressure $p$, is the starting point, together with the metric

$d s^{2}=-e^{\eta(r)} d t^{2}+e^{\epsilon(r)} d r^{2}+r^{2}\left(d \vartheta^{2}+\sin ^{2} \vartheta d \varphi^{2}\right)$,

where the radial metric coefficient takes the form

$$
e^{-\epsilon(r)}=1-\frac{2 m(r)}{r}
$$

One can implement the MGD mappings between Eqs. (53) and (62),

$$
\begin{gathered}
\eta \mapsto \nu=\eta+\zeta g, \\
\epsilon \mapsto \chi=\epsilon-\log (\zeta \kappa) .
\end{gathered}
$$

The $g$ and $\kappa$ scalar fields encode anisotropic effects. The standard MGD procedure can be recovered when one identifies $g=0$ and $\kappa \mapsto \kappa^{*}$, where $\kappa^{*}$ is the MGD function in Eq. (28), with $L=0$, in Sec. II A. Therefore, the time metric component remains the same, whereas the $\theta_{\mu \nu}$ source deforms the radial metric component driven by Eq. (65). When one substitutes the deformed metric into Eqs. (54)-(56), the resulting coupled system of ODEs for $\zeta=0$ reads

$$
\begin{gathered}
\frac{8 \pi G}{c^{4}} \rho=\frac{1}{r^{2}}-e^{-\epsilon}\left(\frac{1}{r^{2}}+\frac{\epsilon^{\prime}}{r}\right), \\
\frac{8 \pi G}{c^{4}} p=-\frac{1}{r^{2}}+e^{-\epsilon}\left(\frac{1}{r^{2}}+\frac{\nu^{\prime}}{r}\right), \\
\frac{8 \pi G}{c^{4}} p=\frac{e^{-\epsilon}}{4}\left(2 \nu^{\prime \prime}+\nu^{\prime 2}+\frac{2 \nu^{\prime}}{r}\right)+\frac{\epsilon^{\prime}}{4} e^{-\epsilon}\left(\nu^{\prime}+\frac{2}{r}\right) .
\end{gathered}
$$

The conservation equation takes the form of Eq. (14), and the part of the coupled system of ODEs that involves $\theta_{\mu \nu}$ is given by

$$
\frac{8 \pi G}{c^{4}} \theta_{0}^{0}=-\frac{\kappa^{* \prime}}{r}-\frac{\kappa^{*}}{r^{2}}
$$

$$
\begin{gathered}
\frac{8 \pi G}{c^{4}} \theta_{1}{ }^{1}=-\kappa^{*}\left(\frac{\nu^{\prime}}{r}+\frac{1}{r^{2}}\right), \\
\frac{32 \pi G}{c^{4}} \theta_{2}{ }^{2}=-\kappa^{*}\left(2 \nu^{\prime \prime}+\nu^{\prime 2}+\frac{2 \nu^{\prime}}{r}\right)-\kappa^{* \prime}\left(\nu^{\prime}+\frac{2}{r}\right) .
\end{gathered}
$$


On the other hand, the conservation equation for $\theta_{\mu \nu}$ reads

$$
\left(\theta_{1}^{1}\right)^{\prime}-\frac{\nu^{\prime}}{2}\left(\theta_{0}^{0}-\theta_{1}^{1}\right)+\frac{2}{r}\left(\theta_{1}^{1}-\theta_{2}^{2}\right)=0
$$

\section{MGD YANG-MILLS-DIRAC STELLAR CONFIGURATIONS: RESULTS, ANALYSIS, AND DISCUSSION}

Compact distributions can be described by MGD. Considering a background fermionic field $\psi$ of mass $m$, MGD stellar configurations can be derived as solutions of Yang-Mills-Einstein-Dirac coupled system field equations. One can take the spin- $1 / 2$ fermionic field minimally coupled to gravity (Einstein-Hilbert) and to the $\mathrm{SU}(2)$ Yang-Mills fields. The action for this system reads [60]

$$
S_{\mathrm{tot}}=S_{\mathrm{EH}}+S_{\psi}+S_{\mathrm{YM}},
$$

where

$$
\begin{gathered}
S_{\mathrm{EH}}=-\frac{c^{3}}{16 \pi G} \int d^{4} x \sqrt{-g} \mathcal{R}, \\
S_{\psi}=\int d^{4} x \mathcal{L}_{\psi}=\int d^{4} x\left[\frac{i \hbar}{2}\left(\bar{\psi} \gamma^{\mu} D_{\mu} \psi-\bar{\psi} \overleftarrow{D}_{\mu} \gamma^{\mu} \psi\right)\right. \\
\left.-m \bar{\psi} \psi+\frac{\lambda}{2 !}(\bar{\psi} \psi)^{2}\right]
\end{gathered}
$$

and $D_{\mu} \psi=\left(\partial_{\mu}+\frac{1}{8} \omega^{a b}{ }_{\mu}\left[\gamma^{a}, \gamma^{b}\right]-\frac{i g}{2} A_{\mu}^{a} \sigma^{a}\right) \psi$, where $\omega_{a b \mu}$ denotes the spin connection. The set $\left\{\gamma^{\mu}\right\}$ is constituted by gamma matrices, satisfying $\left\{\gamma^{\mu}, \gamma^{\nu}\right\}=2 g^{\mu \nu} \mathbb{I}$, and $\bar{\psi}=$ $\psi^{\dagger} \gamma_{0}$ is the spinor conjugate. Dirac matrices in curved spaces, $\gamma^{a}=e_{\mu}^{a} \gamma^{\mu}$, are computed when one employs tetrads $e_{\mu}^{a}$. The term $i \frac{g}{2} A_{\mu}^{a} \sigma^{a} \psi$ describes the coupling between the spin-1/2 fermionic field and the Yang-Mills field, where $g$ is the $\mathrm{SU}(2)$ coupling constant and $\sigma^{a}$ denotes the set of Pauli matrices. In addition $(a, b, c=1,2,3)$,

$$
S_{\mathrm{YM}}=\int d^{4} x \mathcal{L}_{\mathrm{YM}}=-\int d^{4} x \frac{1}{4} F_{\mu \nu}^{c} F^{c \mu \nu},
$$

where $F_{\mu \nu}^{c}=\partial_{[\mu} A_{\nu]}^{c}+\mathrm{g} \epsilon_{a b}^{c} A_{\mu}^{a} A_{\nu}^{b}$ is the Yang-Mills tensor field strength and $A_{\mu}^{a}$ represents the Yang-Mills gauge potential.

With the action (73) and the MGD, one derives the MGD Yang-Mills-Einstein-Dirac system of equations of motion, with MGD decoupling

$$
\begin{gathered}
\mathrm{G}_{\mu \nu}-\Lambda_{b} g_{\mu \nu}-(1+\zeta) \mathcal{T}_{\mu \nu}=\mathfrak{I}_{\mu \nu}, \\
{\left[i \hbar \gamma^{\mu} D_{\mu}-m c \mathbb{I}+\lambda(\mathbb{I}-\bar{\psi} \psi)\right] \psi=0,} \\
\bar{\psi}\left[i \hbar \overleftarrow{D}_{\mu} \gamma^{\mu}+m c \mathbb{I}+\lambda(\mathbb{I}-\bar{\psi} \psi)\right]=0,
\end{gathered}
$$

$\frac{1}{\sqrt{-g}} \partial_{\nu}\left(\sqrt{-g} F^{a \mu \nu}\right)+g \epsilon_{b c}^{a} A_{\nu}^{b} F^{c \mu \nu}=\frac{g \hbar c}{2} \bar{\psi} \gamma^{\mu} \sigma^{a} \psi$

where $\zeta(\sigma) \simeq \sigma^{-1}$ governs the MGD decoupling. The spin$1 / 2$ fermionic stress-energy tensor is given by

$$
\begin{aligned}
\mathcal{T}_{\mu \nu}= & i \hbar g_{\nu}{ }^{\tau} \bar{\psi}\left(\gamma_{(\mu} D_{\tau)}+\overleftarrow{D}_{(\mu} \gamma_{\tau)}\right) \psi-2 \delta_{\mu \nu} \lambda(\bar{\psi} \psi)^{2} \\
& -4 F_{\mu \tau}^{a} F_{\nu}^{a \tau}+\delta_{\mu \nu} F_{\alpha \beta}^{a} F^{a \alpha \beta} .
\end{aligned}
$$

In addition, owing to the tiny phenomenological bound for the term $\mathfrak{l}$ in Eq. (29) [19] that compounds the MGD metric component (44b), the off-diagonal components of Eq. (78) are equal to zero.

The Yang-Mills field has the usual form, emulating a non-Abelian monopole,

$$
A_{i}^{c}=\frac{1-f(r)}{g}\left(\begin{array}{ccc}
0 & \sin \varphi & \frac{\sin (2 \vartheta)}{2} \cos \varphi \\
0 & -\cos \varphi & \frac{\sin (2 \vartheta)}{2} \sin \varphi \\
0 & 0 & -\sin ^{2} \vartheta
\end{array}\right),
$$

with its temporal component being equal to zero.

The following two spinor Ansätze,

$$
\begin{aligned}
& \psi_{1}(r, t)=2 \exp \left(-i \frac{E t}{\hbar}\right)\left(\begin{array}{c}
0 \\
\alpha_{1}(r) \\
i \alpha_{2}(r) \sin \vartheta e^{-i \varphi} \\
-i \alpha_{2}(r) \cos \vartheta
\end{array}\right), \\
& \psi_{2}(r, t)=-2 \exp \left(-i \frac{E t}{\hbar}\right)\left(\begin{array}{c}
\alpha_{1}(r) \\
0 \\
i \alpha_{2}(r) \cos \vartheta \\
i \alpha_{2}(r) \sin \vartheta e^{i \varphi}
\end{array}\right),
\end{aligned}
$$

where $\alpha_{1}, \alpha_{2}:[0, \infty) \rightarrow \mathbb{R}$, were proposed to study coupled systems $[58,59]$.

Substituting Eqs. (80a) and (80b) into the MGD YangMills-Einstein-Dirac system of equations of motion (77a)(77d), the coupled system of ODEs is obtained in terms of the MGD metric (8). For the analysis that follows, one denotes the dimensionless radius, $x=\frac{m r}{\hbar}$ and $\xi=\frac{\pi m}{\hbar c g^{2} M_{p}^{2}}$ [and by " $(\cdot)^{\prime \prime}$ " the derivative with respect to $x$ ], with $M_{p}=$ $\sqrt{\hbar c / G} \approx 1.2209 \times 10^{22} \mathrm{MeV} / c^{2}=2.176 \times 10^{-8} \mathrm{~kg}$ being the Planck mass, and the following rescaled quantities [59,60]:

$$
\begin{gathered}
E \mapsto \tilde{E}=\frac{E}{m c^{2}}, \quad \mathrm{~g} \mapsto \tilde{\mathrm{g}}=\mathrm{g} \sqrt{\hbar c}, \\
M \mapsto \tilde{M}=\frac{m M}{M_{p}^{2}}, \quad \lambda \mapsto \tilde{\lambda}=\frac{\lambda m^{2} c}{4 \tilde{g}^{2} \hbar^{3}}, \\
\alpha_{1,2} \mapsto \tilde{\alpha}_{1,2}=2\left(\frac{m c}{\hbar}\right)^{\frac{3}{2}} \tilde{g} \alpha_{1,2} .
\end{gathered}
$$


Hence, the MGD Yang-Mills-Einstein-Dirac coupled system of ODEs reads, with respect to the metric (8),

$$
\begin{gathered}
\tilde{\alpha}_{2}^{\prime}(x)+\left[\frac{A^{\prime}(x)}{4 \sqrt{A(x) B(x)}}+\frac{1}{x}\right] \tilde{\alpha}_{2}(x)+\frac{f(x) \tilde{\alpha}_{2}(x)}{x \sqrt{B(x)}}+\left[\frac{1}{\sqrt{A(x)}}+8 \lambda \frac{\tilde{\alpha}_{2}^{2}(x)-\tilde{\alpha}_{1}^{2}(x)}{\sqrt{B(x)}}-\frac{\tilde{E}}{\sqrt{A(x) B(x)}}\right] \tilde{\alpha}_{1}(x)=0, \\
\tilde{\alpha}_{1}^{\prime}(x)+\left[\frac{A^{\prime}(x)}{4 \sqrt{A(x) B(x)}}+\frac{1}{x}\right] \tilde{\alpha}_{1}(x)-\frac{f(x) \tilde{\alpha}_{1}(x)}{x \sqrt{B(x)}}+\left[\frac{1}{\sqrt{A(x)}}+8 \tilde{\lambda} \frac{\tilde{\alpha}_{2}^{2}(x)-\tilde{\alpha}_{1}^{2}(x)}{\sqrt{B(x)}}+\frac{\tilde{E}}{\sqrt{A(x) B(x)}}\right] \tilde{\alpha}_{2}(x)=0, \\
\tilde{2} \xi x^{2}\left[\frac{\left(f^{2}(x)-1\right)^{2}}{x^{4}}+\frac{2 \sqrt{A(x) B(x)} f^{\prime 2}(x)}{x^{2}}+\frac{4 \tilde{E}(1+\zeta)}{\sqrt{B(x)}}\left(\tilde{\alpha}_{1}^{2}(x)+\tilde{\alpha}_{2}^{2}(x)\right)+16 \lambda\left(\tilde{\alpha}_{2}^{2}(x)-\tilde{\alpha}_{1}^{2}(x)\right)^{2}\right]=M^{\prime}(x), \\
\frac{8 \xi x}{\sqrt{B(x)}}\left[\frac{\tilde{E}(1+\zeta)}{\sqrt{A(x) B(x)}}\left(\tilde{\alpha}_{1}^{2}(x)+\tilde{\alpha}_{2}^{2}(x)\right)+\tilde{\alpha}_{1}(x) \tilde{\alpha}_{2}^{\prime}(x)-\tilde{\alpha}_{2}(x) \tilde{\alpha}_{1}^{\prime}(x)+\frac{\sqrt{B(x)} f^{\prime 2}(x)}{x^{2}}\right]=0, \\
f^{\prime \prime}(x) \sqrt{A(x) B(x)}+A^{\prime}(x)+\frac{f(x)}{x^{2}}\left(1-f^{2}(x)\right)-2 x \tilde{\alpha}_{1}(x) \tilde{\alpha}_{2}(x)=0 .
\end{gathered}
$$

As Planckian compact stellar distributions are not studied in this work, the fermion mass is considered to be negligible when compared to the Chandrasekhar mass $M_{p}^{3} m^{-2}$. The SU(2) Yang-Mills running constant $g$ is usually inversely proportional to the energy scale of the system. Since the energy scale of compact stellar distributions that interest us has the coupling constant $g$ of the order of unity, $\tilde{\lambda}$ must also be of this order [60].

The MGD of the Schwarzschild metric (8) having temporal and radial coefficients (44a) and (44b) separately satisfies the effective Einstein field equations on the brane (77a) with MGD decoupling. Now we must verify whether the MGD metric and the Ansätze (80a) and (80b), together with the Yang-Mills field (79), satisfy the whole YangMills-Einstein-Dirac coupled system (82a)-(82e). For the system, there will be constraints in the form of the spinor field coefficients $\alpha_{1}(r)$ and $\alpha_{2}(r)$ in Eqs. (80a) and (80b). Integrating the system (82a)-(82e) numerically, one can use boundary conditions with respect to the center of compact distribution [60]:

$$
\begin{aligned}
& \tilde{\alpha}_{1}(x) \approx \alpha_{1 c}+\frac{1}{2 !} \alpha_{1} x^{2}, \quad \tilde{\alpha}_{2}(x) \approx \tilde{\alpha}_{2} x, \\
& \tilde{M}(x) \approx \frac{1}{3 !} \tilde{M}_{3} x^{3}, \quad f(x) \approx 1+\frac{1}{2 !} f_{2} x^{2} .
\end{aligned}
$$

The parameter $\alpha_{1 c}$ indicates $\lim _{x \rightarrow 0} \alpha_{1}(x)$. The coefficients $\alpha_{1}, \alpha_{2}$, and $M_{3}$ in Eqs. (83) and (84) are obtained by the solution and integration of the system (82a)-(82e), whereas $f_{2}, \tilde{E}$, and $\alpha_{1 c}$ are completely arbitrary parameters, but, of course, the values of physical interest must generate regular compact solutions in the asymptotically flat limits $\lim _{x \rightarrow \infty} A(x)=1=\lim _{x \rightarrow \infty} B(x)$ and $\lim _{x \rightarrow \infty} f(x)= \pm 1$ [60]. The ADM mass of the stellar configuration corresponds to $M_{\infty}=\lim _{x \rightarrow \infty} \tilde{M}=\frac{m M}{M_{p}^{2}}$.

Dzhunushaliev and Folomeev $[59,61]$ showed that one can derive stellar configurations with Chandrasekhar mass order for $\tilde{\lambda}<0$ such that $|\tilde{\lambda}| \gg \xi$. It also happens for the MGD Yang-Mills-Dirac stars, where one can take $\tilde{\lambda} \approx 1$ and small values of $\xi$. Otherwise, choosing $\xi \approx 1$ implies Planckian stars. Typical masses $\sim M_{p}^{2} / m$ and radii $\sim m^{-1}$ are regarded for the stellar configurations studied here. For fermion mass $m \approx 1 \mathrm{GeV}$ corresponding to nucleons, the MGD Yang-Mills-Dirac star mass equals $\sim 10^{10} \mathrm{Kg}$, which is much smaller than the Chandrasekhar mass $\sim M_{p}^{3} / m^{2} \gg M_{\odot}$, where $M_{\odot}=1.989 \times 10^{30} \mathrm{Kg}$ denotes the solar mass. On the other hand, for $m \approx 10^{-10} \mathrm{eV}$, the MGD Yang-Mills-Dirac star mass equals $\approx M_{\odot}$ and its radius $\approx 10 \mathrm{~km}$, which is feasible for eventual gravitational wave observations.

After numerical integration, the MGD Yang-Mills-Dirac compact stellar configuration ADM mass parameter, $\tilde{M}_{\infty}=\frac{m M}{M_{\mathrm{p}}^{2}}$, as a function of the central value $\alpha_{1 c}$, has the profile illustrated in Fig. 1 for the MGD-decoupling parameter $\zeta=0.1$. Two different values of the YangMills coupling constant are considered, as well as two different values of the finite brane tension. In fact, as the most precise current bound on the variable brane tension is $\sigma \gtrsim 2.81 \times 10^{6} \mathrm{MeV}^{4}$ [8], Fig. 1 takes this lower brane tension limit and the distinct case $\sigma \sim 10^{12} \mathrm{MeV}^{4}$ to study the physical differences among these cases. It is worth emphasizing that the general-relativistic limit corresponds to a rigid brane, making $\sigma \rightarrow \infty$ and $\zeta \rightarrow 0$.

In Fig. 1, each plot has a peak in the MGD YangMills-Dirac stellar mass at some value of central value $\alpha_{1 c}$ of the spinor field component. For the brane tension 


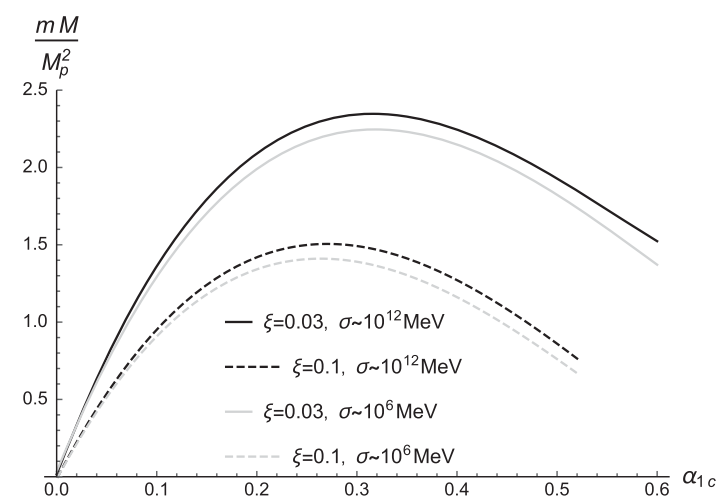

FIG. 1. ADM mass of MGD Yang-Mills-Dirac stars as a function of the central value of the spinor field $\alpha_{1}$ component $\alpha_{1 c}$ for two values $\xi=0.03$ and $\xi=0.1$, and $\tilde{\lambda}=-1$. Two values of the brane tension $\sigma \approx 2.8 \times 10^{6} \mathrm{MeV}^{4}$ and $\sigma \sim 10^{12} \mathrm{MeV}^{4}$ are analyzed for the MGD-decoupling parameter value $\zeta=0.1$.

$\sigma \sim 10^{12} \mathrm{MeV}^{4}$ and $\xi=0.03$, the maximal mass equals $2.32 \tilde{M}_{\infty}$ at $\tilde{\alpha}_{1 c}=0.318$, whereas for $\xi=0.1$ the maximal mass is equal to $1.50 \tilde{M}_{\infty}$ for $\tilde{\alpha}_{1 c}=0.279$. On the other hand, for the most strict bound $\sigma \approx 2.81 \times 10^{6} \mathrm{MeV}^{4}$, when $\xi=0.03$ the maximal mass equals $2.22 \tilde{M}_{\infty}$ at $\tilde{\alpha}_{1 c}=0.332$, whereas for $\xi=0.1$ the maximal mass is $1.40 \tilde{M}_{\infty}$, for $\tilde{\alpha}_{1 c}=0.270$. One realizes that the bigger the brane tension is, the bigger the maximal mass at smaller values of $\tilde{\alpha}_{1 c}$. MGD Dirac stars were studied in a similar context, where a maximal mass was identified to a transition point, splitting stable and unstable MGD Dirac compact stellar configurations [63].

Figure 1 was motivated by the sign of the binding energy, which is defined as the difference between the energy of $\mathrm{n}_{f}$ free particles and the total energy of the system. The number $\mathrm{n}_{f}$ corresponds to a Noether charge, computed when the four-current density $j^{\rho}=\sqrt{-g} \bar{\psi} \gamma^{\rho} \psi$ is taken into account as $\mathrm{n}_{f}=\int_{\mathbb{R}^{3}} j^{0} d^{3} x[60]$, where $j^{0}=r^{2} \sin \vartheta \frac{\left(\psi^{\dagger} \psi\right)}{\sqrt{A}}$. In dimensionless variables,

$$
\mathrm{n}_{f}=\frac{8 \xi M_{p}^{2}}{m^{2}} \int_{0}^{\infty} \frac{\tilde{\alpha}_{1}^{2}(x)+\tilde{\alpha}_{2}^{2}(x)}{\sqrt{A(x)}} x^{2} d x
$$

As in the temporal component (44a) of the MGD metric, the denominator equals the Schwarzschild one, and the particle number in Eq. (85) is the same as in the Schwarzschild metric. As accomplished in Ref. [60], stellar distributions having negative binding energy are unstable. Hence, the plots in Fig. 1 have a range of variables $\alpha_{1 c}$ compatible with positive values of the binding energy. The higher the value of $\xi$ is, the narrower the range of the variable $\alpha_{1 c}$.

Figure 1 also illustrates how the maximal MGD YangMills-Dirac stellar mass increases as a function of the central value $\alpha_{1 c}$ of the spinor component $\alpha_{1}$. For decrements of $\xi$, the maxima of the total ADM mass $M^{\max }$ increase. It is worth emphasizing that Fig. 1 shows the dependence of $M^{\max }$ on $\xi$ for small values of $\xi$ :

$$
M^{\max } \approx \frac{0.403(1+\zeta)}{\sqrt{\xi}} \frac{M_{p}^{2}}{m} .
$$

Outer solutions for the Yang-Mills field $f$ with respect to the dimensionless radius $x$ of the stellar distributions are shown in Fig. 2. Two values, $\xi=0.03$ and $\xi=0.1$, are employed in the analysis for $\alpha_{1 c}=0.15$ and $\tilde{\lambda}=-1$. The brane tension value $\sigma \sim 10^{12} \mathrm{MeV}^{4}$ is adopted, and $\zeta=0.1$ governs the MGD decoupling. Figure 2 illustrates the fine dependence of the Yang-Mills field $f$ on the $\xi$ parameter.

The spinor field components $\alpha_{1}(x)$ and $\alpha_{2}(x)$ as a function of dimensionless radius $x$ are illustrated in Figs. 3 and 4, respectively, for the MGD-decoupling parameters $\zeta=0.01$ and $\zeta=0.1$ and two different values $\xi=0.03$ and $\xi=0.1$, with the central value of the spinor field $\alpha_{1}$ component, $\alpha_{1 c}=0.15$, adopted for $\tilde{\lambda}=-1$. Again, the brane tension value $\sigma \sim 10^{12} \mathrm{MeV}^{4}$ is chosen. The SU(2) Yang-Mills field does not induce significant modifications on the stellar configurations concerning its coupling to the fermionic fields. Therefore, qualitatively the spinor field profiles in Figs. 3 and 4 do not significantly change when compared to the MGD Dirac stellar distributions in Ref. [63] and their GR limit [59].

The $\xi \ll 1$ regime can be further studied. For these values, there is a relation between the spinor field components, asserting that $\tilde{\alpha}_{2} \ll \tilde{\alpha}_{1}$, as seen in Figs. 3 and 4 . Hence, Eq. (82a) yields in this regime

$$
\tilde{\alpha}_{1 \star}=\frac{1}{2 \sqrt{2}} \sqrt{-\left(1-\frac{\tilde{E}}{\sqrt{B}}\right)},
$$

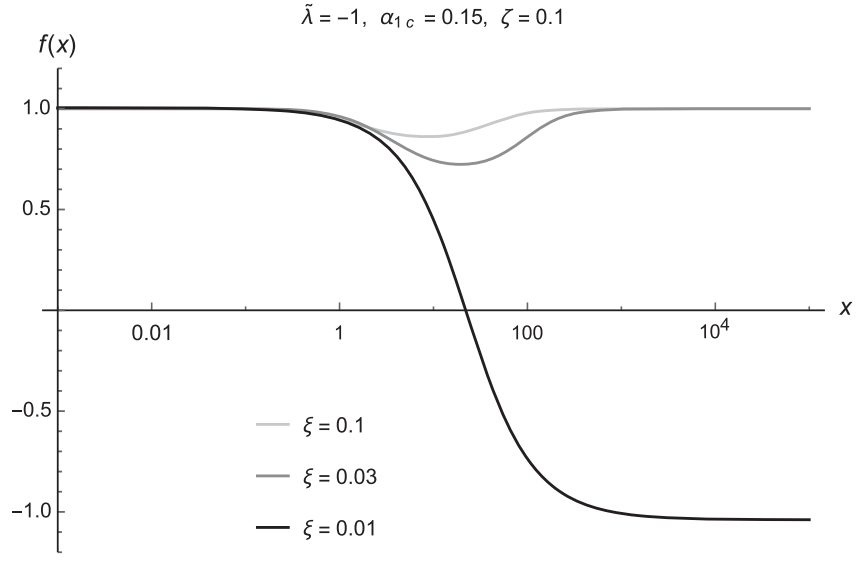

FIG. 2. Linear-log plot of the Yang-Mills field $f(x)$ as a function of dimensionless stellar radius $x$ for $\xi=0.03$ and $\xi=0.1$, with the central value of the spinor field $\alpha_{1}$ component, $\alpha_{1 c}=0.15$, adopted for $\tilde{\lambda}=-1$. The brane tension $\sigma \sim$ $10^{12} \mathrm{MeV}^{4}$ is taken, and $\zeta=0.1$ rules the MGD decoupling. 


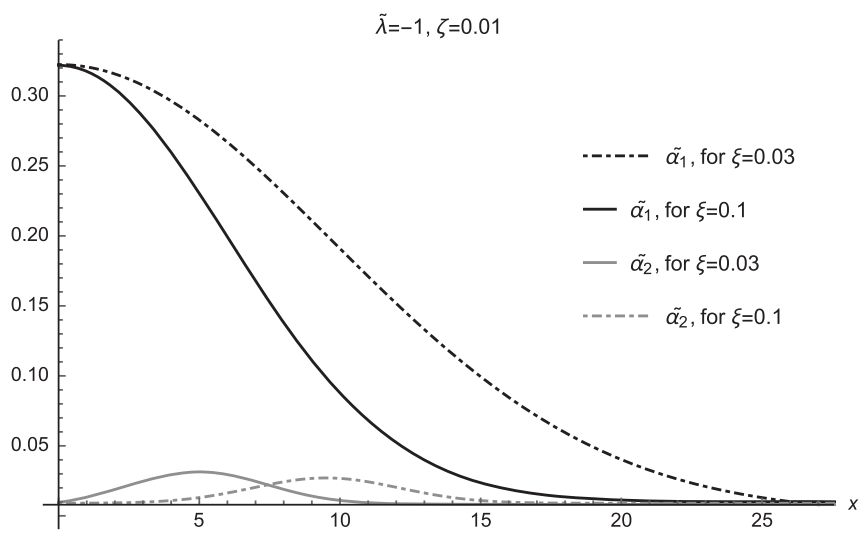

FIG. 3. Spinor field components $\alpha_{1}(x)$ and $\alpha_{2}(x)$ as a function of dimensionless radius $x$ for $\xi=0.03$ and $\xi=0.1$, with the central value $\alpha_{1 c}=0.15$ adopted for $\tilde{\lambda}=-1$. The brane tension $\sigma \sim 10^{12} \mathrm{MeV}^{4}$ is taken and $\zeta=0.01$ rules the MGD decoupling.

where $\tilde{\alpha}_{1 \star}=\sqrt{|\tilde{\lambda}|} \tilde{\alpha}_{1} \quad$ [61]. Substituting Eq. (87) into Eqs. $(82 \mathrm{c})$ and $(82 \mathrm{~d})$, the notation $x_{\star}=\sqrt{\frac{\xi}{|\tilde{\lambda}|}} x$ and $\tilde{M}_{\star}=$ $\sqrt{\frac{\xi}{|\tilde{\lambda}|}} \tilde{M}$ yields

$$
\frac{d \tilde{M}_{\star}}{d x_{\star}}=8 x_{\star}^{2}\left(\frac{\tilde{E}(1+\zeta)}{\sqrt{A}}-4 \tilde{\alpha}_{1 \star}^{2}\right) \tilde{\alpha}_{1 \star}^{2},
$$

Since Eq. (88) lacks the explicit appearance of the parameter $\xi$, one can employ Eq. (88) to determine the ADM (rescaled) mass $\tilde{M}_{\star \infty}=\lim _{x \rightarrow \infty} M_{\star}=\sqrt{\frac{\xi}{|\tilde{\mid}|}} \frac{M_{\star} m}{M_{p}^{2}}$ with respect to $\tilde{E}$. Figure 5 represents the numerical solutions for three different values of the brane tension. For each value of the brane tension, the maximal mass of the MGD YangMills-Dirac stellar configuration is given by



FIG. 4. Spinor field components $\alpha_{1}(x)$ and $\alpha_{2}(x)$ as a function of dimensionless radius $x$ for $\xi=0.03$ and $\xi=0.1$, where $\alpha_{1 c}=$ 0.15 is adopted for $\tilde{\lambda}=-1$. The brane tension $\sigma \sim 10^{12} \mathrm{MeV}^{4}$ is taken, and $\zeta=0.1$ rules the MGD decoupling.



FIG. 5. Dimensionless total ADM mass $M_{\star \infty}$ of MGD YangMills-Dirac stellar configurations as a function of the energy of the stationary part of the spinor fields $\tilde{E}$. Brane tension values $\sigma \sim 10^{12} \mathrm{MeV}^{4}$ (black line), $\sigma \sim 10^{9} \mathrm{MeV}^{4}$ (gray line), and $\sigma \sim$ $10^{6} \mathrm{MeV}^{4}$ (light gray line) are taken, and $\zeta=0.1$ rules the MGD decoupling.

$$
M_{\star}^{\max } \approx 0.403(1+\zeta) \sqrt{\frac{|\tilde{\lambda}|}{\xi}} \frac{M_{p}^{2}}{m}
$$

It is worth emphasizing that when $\tilde{\lambda}=-1$, Eq. (86) is immediately recovered. In addition, our results lead to the ones in the general-relativistic limit in Ref. [60].

The brane tension values $\sigma \sim 10^{12} \mathrm{MeV}^{4}$ (black line in Fig. 5) is nearer the general-relativistic limit, whereas $\sigma \approx 2.81 \times 10^{6} \mathrm{MeV}^{4}$ represents the phenomenological current bound [8], which is more realistic for astrophysical applications. When $\sigma \sim 10^{12} \mathrm{MeV}^{4}$, the maximal mass reads $M_{\star \infty}^{\max }=0.436$ at $\log (\tilde{E})=0.56$; for $\sigma \sim$ $10^{9} \mathrm{MeV}^{4}$, one has $M_{\star \infty}^{\max }=0.419$ at $\log (\tilde{E})=0.54$, and when $\sigma \approx 2.81 \times 10^{6} \mathrm{MeV}^{4}$, the maximal mass is given by $M_{\star \infty}^{\max }=0.402$ at $\log (\tilde{E})=0.59$. The bigger the brane tension is, the bigger the peak corresponding to the stellar maximal mass.

As accomplished with the ADM mass of the MGD YangMills-Dirac stellar configurations, their effective radius can be also studied. For it, the same analog approach will be employed here as that for the GR limit in Refs. [60,74], defining the effective radius of MGD Yang-Mills-Dirac stellar configurations as

$$
\mathrm{R}=\frac{1}{\mathrm{n}_{f}} \int_{\mathbb{R}^{3}} r j^{0} r^{2} \sin \vartheta d r d \Omega=\frac{\hbar}{\mathrm{n}_{f} m c} \int_{0}^{\infty} \frac{\tilde{\alpha}_{1}^{2}+\tilde{\alpha}_{2}^{2}}{\sqrt{A}} x^{3} d x
$$

It depends on the parameter $\xi$ in the regime $\xi \ll 1$ as

$$
\mathrm{R}_{\star}^{\max } \approx 1.078(1+\zeta) \sqrt{\frac{|\tilde{\lambda}|}{\xi}} \frac{\hbar}{m c} .
$$


This result leads to the ones in the general-relativistic limit in Ref. [60].

\section{CONCLUSIONS AND PROSPECTS}

MGD Yang-Mills-Dirac stellar configurations, consisting of nonlinear spinor fields minimally coupled to gravity in an SU(2) Yang-Mills background on a fluid brane with finite tension were studied and discussed. Two feasible fermionic fields Ansätze were employed as compatible spinor solutions to the MGD metric. These spinor fields, which compose the MGD Yang-Mills-Dirac stars, have components plotted in Figs. 3 and 4, respectively, for the MGD-decoupling parameter $\zeta=0.01$ and $\zeta=0.1$ and two different values $\xi=0.03$ and $\xi=0.1$ for a specific value of the central value $\alpha_{1 c}=0.15$. The spinor selfinteraction parameter $\tilde{\lambda}=-1$ was adopted throughout the analysis. In fact, for these values and small values of $\xi$, stellar configurations with mass of the order of the Chandrasekhar mass can be derived, also avoiding Planckian compact MGD Yang-Mills-Dirac stellar configurations. The SU(2) Yang-Mills field was shown not to have a significant influence on the stellar configurations, mainly regarding the coupling to the fermionic fields. However, Fig. 2 reveals a fine dependence of the Yang-Mills field $f$ on the $\xi$ parameter. Hence, the spinor field profiles in Figs. 3 and 4 do not significantly change when compared to the MGD Dirac stellar distributions in Ref. [63] or the GR limit in Ref. [60]. In addition, Fig. 5 showed the total ADM mass of MGD Yang-Mills-Dirac stellar configurations as a function of the energy of the stationary part of the spinor fields. The lower the brane tension is, the lower the peak corresponding to the MGD Yang-Mills-Dirac stellar maximal mass.

The MGD Yang-Mills-Dirac stars are qualitatively similar to their general-relativistic limit counterparts. In fact, the MGD parameter $\mathfrak{l}$ in Eq. (29) attains small values [19]. However, even small, it does affect the solutions found by solving the system (82a)-(82e). The MGD-decoupling parameter $\zeta \sim \sigma^{-1}$ also affects, for instance, the results in Eqs. (77a), (82c), (82d), (86), and (88), making the maximal mass and the maximal radius, respectively, in Eqs. (89) and (91) vary according to the most strict bound for the brane tension $\sigma \gtrsim 2.81 \times 10^{6} \mathrm{MeV}^{4}$ [8]. In the general-relativistic limit, when $\sigma \rightarrow \infty$, and consequently $\zeta \rightarrow 0$, the GR results in Ref. [60] are recovered.

Although relatively similar, from the qualitative point of view, to some results in the GR limit of Ref. [60], the MGD Yang-Mills-Dirac stars' stellar configurations studied here bring a more realistic model, making them more feasible and the eventual gravitational wave observations in LIGO and eLISA more reliable. Indeed, the MGD Yang-MillsDirac stars' stellar configurations take into account a finite brane tension whose current values comply with the CMB anisotropy and also with well established cosmological models. For example, the same procedure implemented in the MGD context in Refs. [21,22], which predicts the highest frequencies of gravitational wave (GW) radiation emitted by MGD star mergers and their GW windows to be experimentally detectable in LIGO and eLISA, can be studied for MGD Yang-Mills-Dirac stars. A very important result is the numerical solution in Fig. 5 for three different values of brane tension. The maximal masses of the MGD Yang-Mills-Dirac stellar configurations, as a function of the energy, are smaller for smaller values of the brane tension. In fact, for any finite value of the brane tension, the MGD Yang-Mills-Dirac stellar configuration maximal mass is smaller than the GR limit studied in Ref. [60]. This difference can be observationally explored in current experiments on GWs determining specific physical signatures of MGD Yang-Mills-Dirac stars.

Beyond the scope of this work, as a relevant perspective, one can add mass to the Yang-Mills gauge potential to generate MGD Proca stars. Their GR limit were studied in Refs. [60,75] with a static, regular, asymptotically flat metric [76]. Current proposals assert that a massive spin-1 gauge field can constitute dark matter. Hence, MGD Proca stars can play an important role in modeling realistic stellar configurations that are in full compliance with current cosmological models. Although the MGD Yang-MillsDirac stellar configurations have been scrutinized here from a first quantized setup, second quantized quantum effects are also worthy of investigation. Spin- $1 / 2$ fermions were used in this work, but higher spin fermions may be also useful to couple to Yang-Mills field and gravity. In addition to the spin-3/2 Rarita-Schwinger field that was proposed in Ref. [62], other fermionic fields, including flagpole, mass dimension 1 spinor fields [77], might serve as a useful Ansätze alternative to the ones in Eqs. (80a) and (80b).

\section{ACKNOWLEDGMENTS}

R. d. R. is grateful to FAPESP (Grant No. 2017/18897-8) and to the National Council for Scientific and Technological Development [(CNPq) Grants No. 303390/2019-0, No. 406134/2018-9, and No. 303293/2015-2] for partial financial support. 
[1] J. Ovalle, Phys. Rev. D 95, 104019 (2017).

[2] J. Ovalle, Int. J. Mod. Phys. D 18, 837 (2009).

[3] J. Ovalle, Mod. Phys. Lett. A 25, 3323 (2010).

[4] R. Casadio and J. Ovalle, Gen. Relativ. Gravit. 46, 1669 (2014).

[5] J. Ovalle, L. Gergely, and R. Casadio, Classical Quantum Gravity 32, 045015 (2015).

[6] I. Antoniadis, N. Arkani-Hamed, S. Dimopoulos, and G. R. Dvali, Phys. Lett. B 436, 257 (1998).

[7] I. Antoniadis, Phys. Lett. B 246, 377 (1990).

[8] A. Fernandes-Silva, A. J. Ferreira-Martins, and R. da Rocha, Phys. Lett. B 791, 323 (2019).

[9] R. Casadio, J. Ovalle, and R. da Rocha, Classical Quantum Gravity 31, 045016 (2014).

[10] J. Ovalle, F. Linares, A. Pasqua, and A. Sotomayor, Classical Quantum Gravity 30, 175019 (2013).

[11] J. Ovalle, Phys. Lett. B 788, 213 (2019).

[12] J. Ovalle and F. Linares, Phys. Rev. D 88, 104026 (2013).

[13] J. Ovalle and A. Sotomayor, Eur. Phys. J. Plus 133, 428 (2018).

[14] M. C. B. Abdalla, J. M. Hoff da Silva, and R. da Rocha, Phys. Rev. D 80, 046003 (2009).

[15] R. da Rocha and J. M. Hoff da Silva, Phys. Rev. D 85, 046009 (2012).

[16] L. A. Gergely, Phys. Rev. D 79, 086007 (2009).

[17] J. Ovalle, Mod. Phys. Lett. A 23, 3247 (2008).

[18] J. Ovalle, R. Casadio, R. da Rocha, A. Sotomayor, and Z. Stuchlik, Eur. Phys. J. C 78, 960 (2018).

[19] R. Casadio, J. Ovalle, and R. da Rocha, Europhys. Lett. 110, 40003 (2015).

[20] R. Casadio and R. da Rocha, Phys. Lett. B 763, 434 (2016).

[21] R. da Rocha, Phys. Rev. D 95, 124017 (2017).

[22] A. Fernandes-Silva, A. J. Ferreira-Martins, and R. da Rocha, Eur. Phys. J. C 78, 631 (2018).

[23] J. Ovalle, R. Casadio, R. da Rocha, A. Sotomayor, and Z. Stuchlik, Europhys. Lett. 124, 20004 (2018).

[24] R. da Rocha, Eur. Phys. J. C 77, 355 (2017).

[25] A. Fernandes-Silva and R. da Rocha, Eur. Phys. J. C 78, 271 (2018).

[26] E. Contreras, Eur. Phys. J. C 78, 678 (2018).

[27] R. Casadio, E. Contreras, J. Ovalle, A. Sotomayor, and Z. Stuchlick, Eur. Phys. J. C 79, 826 (2019).

[28] A. Rincón, L. Gabbanelli, E. Contreras, and F. Tello-Ortiz, Eur. Phys. J. C 79, 873 (2019).

[29] J. Ovalle, C. Posada, and Z. Stuchlík, Classical Quantum Gravity 36, 205010 (2019).

[30] L. Gabbanelli, J. Ovalle, A. Sotomayor, Z. Stuchlik, and R. Casadio, Eur. Phys. J. C 79, 486 (2019).

[31] S. K. Maurya, Eur. Phys. J. C 79, 958 (2019).

[32] F. Tello-Ortiz, S. K. Maurya, A. Errehymy, K. Singh, and M. Daoud, Eur. Phys. J. C 79, 885 (2019).

[33] E. Morales and F. Tello-Ortiz, Eur. Phys. J. C 78, 841 (2018).

[34] E. Morales and F. Tello-Ortiz, Eur. Phys. J. C 78, 618 (2018).

[35] G. Panotopoulos and A. Rincón, Eur. Phys. J. C 78, 851 (2018).

[36] K. Singh, S. K. Maurya, M. K. Jasim, and F. Rahaman, Eur. Phys. J. C 79, 851 (2019).
[37] S. K. Maurya and F. Tello-Ortiz, Eur. Phys. J. C 79, 85 (2019).

[38] J. Ovalle, R. Casadio, R. da Rocha, and A. Sotomayor, Eur. Phys. J. C 78, 122 (2018).

[39] L. Gabbanelli, A. Rincón, and C. Rubio, Eur. Phys. J. C 78, 370 (2018).

[40] R. Pérez Graterol, Eur. Phys. J. Plus 133, 244 (2018).

[41] C. L. Heras and P. Leon, Fortschr. Phys. 66, 1800036 (2018).

[42] V. A. Torres-Sánchez and E. Contreras, Eur. Phys. J. C 79, 829 (2019).

[43] S. Hensh and Z. Stuchlík, Eur. Phys. J. C 79, 834 (2019).

[44] E. Contreras, A. Rincón, and P. Bargueño, Eur. Phys. J. C 79, 216 (2019).

[45] E. Contreras and P. Bargueño, Eur. Phys. J. C 78, 985 (2018).

[46] M. Sharif and S. Sadiq, Eur. Phys. J. C 78, 410 (2018).

[47] C. Stelea, M. A. Dariescu, and C. Dariescu, Phys. Rev. D 97, 104059 (2018).

[48] S. K. Maurya and F. Tello-Ortiz, Phys. Dark Universe 27, 100442 (2020).

[49] F. X. Linares Cedeno and E. Contreras, Phys. Dark Universe 28, 100543 (2020).

[50] D. Deb, M. Khlopov, F. Rahaman, S. Ray, and B. K. Guha, Eur. Phys. J. C 78, 465 (2018).

[51] M. Estrada, Eur. Phys. J. C 79, 918 (2019).

[52] M. Sharif and S. Saba, Eur. Phys. J. C 78, 921 (2018).

[53] E. Contreras and P. Bargueño, Eur. Phys. J. C 78, 558 (2018).

[54] E. Contreras, Classical Quantum Gravity 36, 095004 (2019).

[55] E. Contreras and P. Bargueño, Classical Quantum Gravity 36, 215009 (2019).

[56] M. Sharif and S. Sadiq, Chin. J. Phys. (Taipei) 60, 279 (2019).

[57] R. da Rocha and A. A. Tomaz, Eur. Phys. J. C 79, 1035 (2019).

[58] C. A. R. Herdeiro, A. M. Pombo, and E. Radu, Phys. Lett. B 773, 654 (2017).

[59] V. Dzhunushaliev and V. Folomeev, Phys. Rev. D 99, 084030 (2019).

[60] V. Dzhunushaliev and V. Folomeev, Phys. Rev. D 101, 024023 (2020).

[61] V. Dzhunushaliev and V. Folomeev, Phys. Rev. D 99, 104066 (2019).

[62] C. Herdeiro, I. Perapechka, E. Radu, and Y. Shnir, Phys. Lett. B 797, 134845 (2019).

[63] R. da Rocha, Symmetry 12, 508 (2020).

[64] K. A. Bronnikov, Yu. P. Rybakov, and B. Saha, Eur. Phys. J. Plus 135, 124 (2020).

[65] B. Saha, Can. J. Phys. 94, 116 (2016).

[66] L. Fabbri, Gen. Relativ. Gravit. 47, 1837 (2015).

[67] L. Fabbri, S. Vignolo, and S. Carloni, Phys. Rev. D 90, 024012 (2014).

[68] R. Bartnik and J. Mckinnon, Phys. Rev. Lett. 61, 141 (1988).

[69] T. Shiromizu, K. i. Maeda, and M. Sasaki, Phys. Rev. D 62, 024012 (2000).

[70] T. Shiromizu and D. Ida, Phys. Rev. D 64, 044015 (2001). 
[71] R. Maartens and K. Koyama, Living Rev. Relativity 13, 5 (2010).

[72] J. Ovalle, R. Casadio, and A. Sotomayor, arXiv:1612 .07926.

[73] R. T. Cavalcanti, A. Goncalves da Silva, and R. da Rocha, Classical Quantum Gravity 33, 215007 (2016).
[74] P. Jetzer and J. J. van der Bij, Phys. Lett. B 227, 341 (1989).

[75] M. Minamitsuji, Phys. Rev. D 96, 044017 (2017).

[76] R. Brito, V. Cardoso, C. A. R. Herdeiro, and E. Radu, Phys. Lett. B 752, 291 (2016).

[77] R. da Rocha and W. A. Rodrigues, Jr., Mod. Phys. Lett. A 21, 65 (2006). 